\title{
Dietitian management of oral nutritional supplement prescribing is cost effective and improves quality of care
}

\author{
R. A. Skinner and J. C. Smith \\ Newcastle Nutrition, Royal Victoria Infirmary, Newcastle upon Tyne NE1 4LP, part of Newcastle upon Tyne Hospitals NHS \\ Foundation Trust
}

National Institute for Health and Clinical Excellence clinical guidelines ${ }^{(1)}$ advise that when oral nutritional support is required to treat malnutrition, this should be individualised to provide balanced nutrition. Patients should be monitored and the nutrition support stopped if patients are able to achieve adequate intake from normal food. Dietary advice to boost intake is recommended as the first line in nutrition support; however evidence for this type of intervention is weak ${ }^{(2)}$. It is also a contentious issue as to whether routine prescription of oral nutritional supplements (ONS) is more effective than dietary advice alone ${ }^{(3)}$. Dietitians have the expertise to carry out nutritional assessment, tailor dietary advice to individual requirements and advise on ONS when required. Following concerns regarding spiralling prescribing costs of ONS and general uncertainty regarding how to approach managing malnourished patients, a one-year project was set up to evaluate the effects of a dietitian-led service to review patients requiring ONS. Key objectives included auditing present management of malnourished patients, reducing any inappropriate prescribing of ONS and assessing whether a system of dietitian review is costeffective. A total of 180 patients ( $78 \%$ over 60 years of age) from twenty-eight practices in Newcastle upon Tyne, were reviewed. At the time of the review ninety-eight patients $(54 \%)$ had been taking the products for 12 months or longer.

When ONS were initiated, only fifty-nine patients $(33 \%)$ had weight recorded and twenty-eight patients (15\%) had some objectives or plans for monitoring outlined in GP records. Apart from twenty-five patients who had seen a dietitian, only seven other patients were shown in the documentation to have received any dietary advice in addition to the prescription of ONS. Seventy patients (39\%) did not appear to have clear instructions on how to take the products regularly e.g. prescribed 'as directed' or 'as required' and ninety patients $(50 \%)$ were not collecting scripts regularly.

BMI was calculated to assess current nutritional status for 136 of the 180 patients reviewed during the project. Fifty patients (37\%) were underweight, seventy-nine (58\%) were a healthy weight or overweight. Seven patients $(5 \%)$ were obese all of whom expressed a desire to lose weight. At dietetic review, 115 patients (64\%) reported taking supplements in limited amounts i.e. one carton per day or fewer. Dietary review also found fifty-nine patients (33\%) were using low-fat milk and/or following a reduced fat/energy diet. This appeared to be related to fear of increasing their cholesterol level. Following nutritional assessment, a more nutrient-dense diet was advised where required and reassurance given.

Eighty patients $(44 \%)$ continued to require ONS, twenty-four of whom needed to increase the amount prescribed or taken. A total of 100 patients $(56 \%)$ had ONS stopped following the review, eleven of whom were advised to replace them with a micronutrient supplement.

The project has shown that undernourished adults are often not receiving appropriate dietary advice or monitoring. ONS are frequently not prescribed or taken appropriately and prescriptions have been continued without follow up, resulting in wasteful prescribing. Any cost changes from supplements being increased or decreased were extrapolated over a 12-month period resulting in a potential cost saving of $£ 80$ 286. When patients receive nutritional assessment and individual advice on diet and use of ONS from a dietitian, inappropriate prescribing is minimised and this intervention appears to be cost effective.

1. National Institute for Health and Clinical Excellence NICE (2006) Nutrition Support in adults, clinical guideline 32. London: NICE.

2. Stratton RJ (2005) Proc Nutr Soc 64, 325-333.

3. Baldwin C, Parsons T \& Logan S (2007) Cochrane Database of Systematic Reviews 1. Art. No. CD002008. 